Patrizia Farinelli

\title{
Regard critique sur une période sans but : Antigone de Valeria Parrella
}

\begin{abstract}
Mots-clés : Antigone, actualisation d'un mythe, épistémologie, sémiotique du regard
\end{abstract}

DOI: $10.4312 /$ ars.9.1.129-137

\section{Actualité du mythe}

Seul un petit nombre de mythes classiques ont retenu autant l'attention que celui d'Antigone. Pendant l'Idéalisme et tout le siècle suivant, l'intérêt pour la version de Sophocle de ce mythe - et plus généralement pour la tragédie classique - s'explique, selon George Steiner, par une conception tragique de la condition humaine (Steiner, 1984, 2). Quelles seraient alors les raisons de cet intérêt à une époque plus récente, sujette à une perte du sens du tragique ? Car, même durant les soixante dernières années, l'histoire de la fille d'Edipe a été revisitée avec une fréquence surprenante. Selon Hannes Koch $(1992,23)$, auteur d'une étude sur la réception de la figure d'Antigone dans la littérature allemande du $\mathrm{XX}^{\mathrm{e}}$ siècle, la mise en valeur de ce personnage est liée à un moment où les droits de l'homme sont ignorés par le pouvoir. Et ce phénomène de mise en valeur implique toujours une crispation dans la sphère politique ayant pour conséquence de souligner justement l'importance politique du geste d'Antigone.

En effet, dans les nombreuses reprises de cette tragédie au cours du $\mathrm{XX}^{\mathrm{e}}$ siècle, $\mathrm{y}$ compris celle de Brecht (1948) et de certains écrivains des pays de l'ancien bloc esteuropéen - telles que la version du slovène Dominik Smole (1960) et celles de plusieurs auteurs tchèques (Peter Karvaš, Claus Hubalek, Milan Uhde) ${ }^{1}$ - la figure d'Antigone devient une métaphore de la dissidence au sein des systèmes politique centralisés et totalitaires (Koch, 1992, 115-117). Cependant, trouver une raison précise qui soit à même d'expliquer cet intérêt persistant pour ce sujet est aléatoire. Il faut se demander dans quelles périodes les droits de l'homme n'ont pas été respectés et dans quelle mesure le critère politique couvre entièrement les versions de ce mythe apparues au cours du siècle dernier: on pense ici, par exemple, à la version théâtrale de Jean Anouilh (1942), qui se fonde plutôt sur des instances psychologiques et existentielles,

1 Peter Karvaš, Antigona a ti druzi (1962) ; Claus Hubalek, Hrdinve v Thébah nebydilî (1962) ; Milan Uhde, Děvka z města Théby (1967). 
ou à celle du Living theatre (1967), liée aux instances de la révolte antiautoritaire des années soixante, ou encore au travail philosophique et théâtral de María Zambrano, $\mathrm{La}$ tumba de Antígona (1967), qui délivre des réflexions sur l'identité féminine et examine le mythe en fonction d'un renouvellement intérieur du sujet.

Sans prétendre donc établir de façon rigide des critères qui expliqueraient l'intérêt pour le mythe d'Antigone - et spécifiquement pour la version de Sophocle - dans la dernière phase de l'époque moderne, il faut au moins constater le caractère inépuisable de son discours. Pour reprendre un critère cher aux formalistes - c'est-à-dire, la corrélation entre les éléments constitutifs d'un texte comme garantie de son unité et en même temps de sa qualité (Škovskij, 1982, 17) -, on pourrait dire que la densité des relations, que le texte de Sophocle renferme dans un système d'oppositions et de correspondances, entraîne et justifie ses nombreuses réécritures ${ }^{2}$. Si le thème du conflit entre la voix intérieure de l'individu et la loi d'État constitue le point le plus évident et suggère, en premier lieu, des lectures politiques, la configuration complexe de ce conflit en ouvre également d'autres : anthropologiques, éthiques, psychologiques. Il s'agit d'une opposition entre idéalisme et pragmatisme, entre valeurs de la compassion et de la justice, d'un contraste d'opinion sur les raisons de la vie et de la mort, d'un conflit entre les sexes et les générations. C'est, du reste, le propre du langage figuratif - et les mythes le possèdent par excellence - d'être doté d'une sémantique plurielle inconnue au discours logique et strictement argumentatif. La philosophie est prête à reconnaitre la fonction épistémologique et le plein potentiel heuristique de la pensée qui puise dans les mythes, comme le témoigne le débat philosophique sur ce sujet, développé au cours des quarante dernières années, qui considère la mythologie comme 'forme de raison' et non pas comme son opposition ${ }^{3}$.

\section{Actualisation socio-politique dans l'Antigone de Parrella}

Ici, on va envisager une récente réécriture italienne de la tragédie de Sophocle, publiée en 2012 et mise en scène par une compagnie théâtrale de Naples (« Teatro stabile », mise en scène de Luca de Fusco) avant d'être représentée dans plusieurs villes italiennes et aussi à Paris (au Théâtre National de Chaillot). L'auteur, Valeria Parrella, pratique, comme beaucoup de jeunes écrivains contemporains, des formes d'écriture alternatives à celles d'un postmodernisme aujourd'hui déjà épuisé. Ses textes sont dignes d'intérêt soit en raison d'un certain travail sur la langue visant à obtenir un effet

2 Tandis que, selon Paul Ricœur, la disponibilité des mythes, en général, pour de nouveaux emplois dans de nouveaux contextes culturels est due au surplus de sens qui vient de leur richesse symbolique (Ricœur, 2011).

Voir, par exemple : Kolakowsky, 1973 ; Blumenberg, 1979. 
d'étonnement, soit en raison d'une construction discursive impliquant l'utilisation de rupture du fil narratif. Ses textes sont en outre dénués de tout pathétique ${ }^{4}$. Une attention portée à la société contemporaine, toujours présente dans ses œuvres, apparaît aussi dans sa réécriture de la tragédie de Sophocle. Mais avant de considérer la sémantique de cette reprise, voici tout d'abord quelques mots sur l'architecture du texte et sur sa dimension temporelle.

Pour son Antigone, Parrella suit d'assez près la structure de la tragédie de Sophocle sans pour autant se priver d'introduire de petites transformations liées à la conception qui soutient son interprétation. Le texte est divisé en cinq épisodes, précédés d'un prologue ainsi que d'un parodos et conclu par un exode, qui est à son tour suivi d'une prétendue lettre d'Antigone à Hémon. Les épisodes sont séparés par les stasima où, contre toute attente, n'apparaît jamais le chœur mais seulement les coryphées (une femme et un homme qui sont parfois, dans le dialogue, entourés d'autres personnages). La stratégie vise à représenter les faits dans un espace temporel couvrant à la fois le passé et le présent, ce qui souligne le choix de l'auteur de ne pas contourner les possibles significations supra-temporelles du texte. Cette finalité est poursuivie aussi par une dissonance du registre linguistique : d'un langage plutôt soutenu, on tombe parfois, délibérément, dans le registre familier d'aujourd'hui. Par exemple, le Législateur, dans un dialogue avec Antigone (qui venait juste de dire: «Vie et mort sont dignes lorsqu'on peut les conduire de manière indépendante ») banalise les argumentations de celle-ci et, d'un ton irrévérencieux, à travers une formule du langage parlé, lui dit: « Blah, blah, blah, qui te suggère ces définitions ?» (Parrella, 2012, Episodio II, 38) ${ }^{5}$.

L'imaginaire mortuaire qui, dans les versions précédentes de ce mythe restait ancré dans le motif de l'enterrement refusé est transposé ici au champ médical. L'acte de la jeune fille de donner une sépulture à Polynice devient dans ce cas le choix de l'euthanasie. L'Antigone de Parrella refuse pour son frère un traitement médical intensif sur un lit d'hôpital tel que le prescrit la loi, ainsi que de rester elle-même en vie, en prison, avec l'aide d'une thérapie sédative et dans un état d' "invisibilité » (Parrella, 2012, Episodio V, 89). L'attention portée au monde contemporain ne se limite pas, cependant, à quelques débats animés au cours des dernières années tout particulièrement en Italie (ceux sur l'euthanasie ${ }^{6}$ et les conditions de vie inhumaines dans les prisons) ; elle émerge d'abord dans une vision critique d'une époque qui

4 Parrella a écrit des récueils de contes (Mosca più balena, 2003, Per grazia ricevuta, 2005), des romans (Lo spazio bianco, 2008, Ma quale amore, 2010, Lettera di dimissioni, 2011, Tempo di imparare, 2013) et des pièces pour le théâtre.

5 Le livre de Parrella n'étant pas encore paru en français, je cite certains passages du texte d'après ma propre traduction.

6 Le cas d'Eluana Englaro, en 2009, et d'autres cas analogues ont suscité en Italie dans les dernières années un vif débat sur le thème de l'euthanasie. 
semble être sans issue et va également aborder la question d'une opinion publique souvent indifférente. Le véritable adversaire d'Antigone n'est alors pas, dans ce cas, le Législateur (suppléant de Créon), mais plutôt une communauté passive.

Dans le premier stasimon, dans le dialogue entre les coryphées, on souligne que la douleur de cette époque vient de son être « sans but ( Parrella, 2012, Stasimo I, 27). Les coryphées parlent maintenant seulement en leur propre nom. L'absence du chœur dans le texte, et donc d'une voix capable d'exprimer une conscience collective, fait allusion à une condition historique d'immobilité. Le coryphée et la coryphée s'interrogent justement sur cette absence et sur ce qu'est devenue leur fonction de porte-parole de la communauté ; ils savent qu'ils ne peuvent parler maintenant qu'en tant qu'individus.

L'acte d'Antigone d'arracher les intubations qui maintiennent Polynice dans un état végétatif, n'est pas seulement une rébellion contre quelque chose qui n'est plus véritablement une vie, il devient aussi le signe d'un nécessaire ébranlement que l'individu est appelé à donner à l'histoire. Dans cet appel à la responsabilité, la lecture supra-historique du mythe, encore sauvegardée, assume en même temps une signification historique précise. Cela est souligné par de nombreux passages du texte et, parmi eux, par la lettre d'Antigone à son bien aimé. La jeune fille, qui a déjà dit adieu au monde, regarde de loin une ville (synecdoque de ce monde) qui est une sorte de labyrinthe sans base solide:

[...] Dans mon vol nocturne je vois la ville d'en haut, je sais que tu es quelque part ici-bas. Mais la ville se plaint dans la nuit de soi-même, Hémon, parce que c'est une nuit sombre et sans rêves. Ainsi les escaliers qui montent s'interrompent sans fin, les tours qui se dressent n'ont pas de fondations, les maisons manquent de portes et fenêtres, les rues se croisent sans solution, les murs s'effritent et des plafonds il pleut des cordes, même si le temps n'est pas maussade (Parrella, 2012, La lettera, 97).

Face à ce spectacle surréel, Antigone souhaite la présence de quelqu'un qui sache rêver, parce que c'est seulement dans le rêve, dit-elle, qu'un nouveau départ sera possible. Dans son désir d'un élan de courage, on pourrait de même entrevoir, à côté d'une exhortation sociopolitique, une allusion à la littérature pour sa capacité d'imaginer d'autres mondes. De cette façon, l'auteur revient sur ce potentiel utopique, cette propriété que Johann Gottfried Herder assigne aux mythes (Cometa, 1989, 74-85).

\section{Sémiotique du regard et du mouvement}

L'inhumanité de la loi transgressée par Antigone dans la version de Parrella se trouve dans le fait d'imposer une condition de vie végétative, soumise à un pouvoir 
moins visible mais incisif, exercé, comme le soulignait Foucault, à travers le contrôle du corps. L'héroïne refuse une existence dépourvue de l'élément qui la rendrait humaine (elle entend par « humaine » une vie où l'homme a la possibilité de rechercher le sens des choses). Elle explicite qu'elle, contrairement à ceux qui regardent le spectacle de sa condamnation avec une sorte de voyeurisme et qui de Polynice n'avaient « rien vu [...] ", elle, en revanche, a vu de très près, pendant longtemps, la non-vie de son frère et n'a pas pu rester indifférente face à cette impuissance.

Giacomo Debenedetti nous rappelle (dans un contexte tout à fait différent) un processus typique de la tragédie : «[...] où un protagoniste est régulièrement contraint par les événements à ouvrir les yeux et à prendre conscience de son destin [...] » (Debenedetti, 1970, 102). Cela est bien présent dans ce texte où le conflit entre le moi et la communauté se manifeste par l'acte de regarder. La sémiotique du regard absent ou présent revient dans plusieurs passages et se charge de significations différentes. Les paupières de Polynice dans une salle à la lumière artificielle ne sont pas seulement, dans cette reprise du mythe, celles d'un homme dans un état prolongé de coma ; car, au sens figuré, cette image montre également la manière d'être d'une communauté politiquement inactive, qui décline toutes décisions et responsabilités. Contrairement à ceux qui montrent de l'indifférence, Antigone revendique sa capacité de voir. (Elle agit parce qu'elle a vu.) Et ce n'est pas un hasard si, en prison, elle déplore sa nouvelle condition d'invisibilité. Dans le prologue, elle exige le regard attentif du public : « Je veux que vous tous soyez éveillés, ayez les pupilles dilatées, une attention soutenue, vive » : un appel, celui-ci, qui va bien au-delà de sa fonction traditionnelle d'inviter le public à suivre avec intérêt l'histoire représentée et devient ainsi la devise de la conception qui soutient cette revisitation du mythe, c'est-à-dire la nécessité d'intervenir, en tant qu'individus et communauté, à la sauvegarde du sens de la vie pour retrouver en même temps le sens de l'histoire. Des yeux ouverts, donc, comme image d'une existence active et comme issue pour sortir d'un état historique de torpeur (Parrella, 2012, Stasimo III, 54).

La sémiotique du mouvement physique, faisant allusion à une pensée privée de rigidité, est non moins significative. L'opposition entre dogmatisme et flexibilité mentale, respectivement visualisés par le Législateur et par Antigone, s'annonce comme une opposition à une manière spécifique d'être dans le monde et de voir l'histoire. L'immobilité du Législateur ne lui permet ni de comprendre la différence entre justice et vérité ni d'avoir des doutes. À la rigidité mentale de l'homme au pouvoir, s'ajoute ici une société qui ne trouve pas un telos et qui est en train de sombrer. Antigone, au contraire, se profile dans la mobilité ${ }^{7}$ Elle-même, en parlant de soi, admet - avec une certitude qui me semble étonnante par rapport aux paradigmes épistémologique et

7 Le personnage de Tiresias définit les événements de la façon suivante : «Ce qui s'est passé, s'est passé, parce qu'Antigone est le mouvement [...]» (Parrella 2012, Episodio IV, p. 73). 
axiologique de notre époque - qu'elle procède "selon une ligne droite " (Parrella, 2012, Episodio V, 85).

\section{Contre une pensée de l'ambivalence}

Cette réécriture de la tragédie antique semble vouloir couper tout lien avec la pensée de l'ambivalence, du doute, de l'indicible. Cela se voit en particulier dans les stratégies choisies pour la construction des personnages et de la langue.

L'auteur prétend que son Antigone est consciente de la grande portée de son geste de dissidence, un geste accompli bien sûr en raison d'un cas singulier et de l'affect parental, mais en même temps d'un sens de responsabilité envers tous. Derrière ses mots on retrouve parfois même la figure du Christ : « Ma loi intérieure va devenir dans un instant extérieure et versée en sacrifice pour vous » (Parrella, 2012, Prologo, 8). La création d'un personnage représentant quelqu'un capable d'une action décisive, bien conscient de son rôle, nous invite, alors, à réexaminer le statut du personnage littéraire d'aujourd'hui. Il faut s'interroger si le " personnage ", tel qu'il apparaît tout au long d'un siècle et représentant un sujet dominé par l'incertitude et agissant presque toujours par hasard, pourrait appartenir désormais au passé. On pense ici, par exemple, à la très humaine Antigone de Jean Anouilh, qui était prise par des doutes et ne savait même plus, à la fin, pour qui elle avait agi, si c'était pour son frère ou pour elle-même ou à cause d'autres raisons inexplicables. L'héroïne de Parrella, en revanche, n'est jamais frappée par l'indécision, par des doutes au sujet de la vérité. La figure du Législateur est elle aussi découpée d'une manière univoque : il devient ainsi la caricature d'un homme misérable, dépourvu de la capacité d'argumenter d'une manière convaincante ; ses faibles prises de position se traduisent aussi par son regret de n'avoir pas signé à temps un acte d'amnistie.

Dans les œuvres précédentes de cet auteur, on trouvait parfois la stratégie de l'espace blanc ou bien la présence d'un discours interrompu, capable de donner une signification aux faits (et souvent une signification plurielle) d'une manière moins directe. Le lecteur averti aurait donc pu s'attendre à ce que - dans Antigone aussi quelques mots restent dans l'espace interstitiel et que les positions et les sentiments, dont est chargé le personnage principal, se délivrent plutôt dans le non-dit. Cette attente était renforcée par une auto-exhortation d'Antigone, au début de son discours, à utiliser ses mots de manière avare: «Par la parole incomplète doit s'élever la vérité, si mon action apporte la vérité [...].» Et encore: « Après, Antigone, tais-toi, si tu peux, tais-toi. » (Parrella, 2012, Prologo, 8-9) Cependant, ni les attentes du lecteur ni les intentions préliminaires de la protagoniste ne se réalisent. Son discours est soutenu, plein de dignité, souvent sentencieux, non pas retenu ou brisé. 
Pour conclure, l'appel présent dans ce texte à retrouver le sens de la responsabilité a une indéniable pertinence éthique et sociopolitique ; en outre, cette actualisation du mythe a le mérite de conserver en même temps la possibilité d'une lecture supratemporelle. Restent plutôt sujets à caution le fort concept de vérité qui imprègne le discours et les modalités trop explicites par lesquels l'appel d'Antigone se formule. Les personnages sont ainsi simplifiés, les mots réduits au pur moyen de communication. On pourrait pourtant attendre d'un texte littéraire qu'il soit, en plus, une invitation à une réflexion sur la complexité.

\section{Bibliographie}

Anouilh, J., Antigone, Paris [1948] 2008.

Bignotti, S., Maria Zambrano: Il mito di Antigone, in : Attualità del mito (éd. Grassi, P.), numéro spécial de Hermeneutica. Annuario di Filosofia e teologia 30 n. s., 2011, p. 363-395.

Blumenberg, H., Arbeit am Mythos, Francfort 1979.

Cometa, M., Um also zu träumen, seyd nüchtern. Mitologie della ragione in J. G. Herder, in: Mitologie della ragione. Letterature e miti dal Romanticismo al moderno (éd. Fuhrman, M.), Pordenone 1989, p. 31-93.

Debenedetti, G., Il romanzo del Novecento, Milan 1970.

Fuhrman, M., Mythos als Wiederholung, in: Terror und Spiel. Probleme der Mythenrezeption : Poetik und Hermeneutik, 4 (éd. Fuhrman, M.), Munich 1971, p. 121-143.

Kolakowsky, L., Die Gegenwärtigkeit des Mythos, Munich, Zurich 1973.

Koch, H., Auf den Spuren der Antigone. Die Gestalt der Antigone in der deutschen Literatur des zwanzigsten Jahrhunderts, Trieste 1992.

Parrella, V., Antigone, Turin 2012.

Sofocle, Antigone, Edipo re, Edipo a Colono (éd. et trad. Ferrari, F.), Milan $1987^{3}$.

Ricoeur, P., Le symbole et le mythe, Bullettin du centre Protestant d'Études, 6, 1963, p. 14-19; trad. it. Bertoletti, I., Il simbolo e il mito, in : Attualità del mito (éd. Grassi, P.), numéro spécial de Hermeneutica. Annuario di Filosofia e teologia 30 n. s., 2011, p. 417-424.

Steiner, G., Antigones, Oxford 1984 ; trad. it. Marini, N., Le Antigoni, Milan $2003^{2}$.

Steiner, G., The Death of tragedy, Londres 1961 ; trad. it. Scudder, G., Morte della tragedia, Milan $2005^{3}$.

Šklovskij, V., Tetiva. O nešodstve šodnogo, Moskva 1970 ; trad. it. Klein, E., Simili e dissimile. Saggi di poetica, Milan 1982. 


\section{S kritičnim pogledom na brezcilino obdobje: Antigone Valerie Parrella}

Ključne besede: Antigona, aktualizacija mita, epistemologija, semiotika pogleda, odgovornost

Gostota motivov, ki jih predstavlja mit o Antigoni, bi lahko že sama po sebi pojasnila razlog za tolikšno število interpretacij in aktualizacij. V njih je konflikt med notranjim glasom posameznika in zakonom Države postavljen $\mathrm{v}$ sámo središče in $\mathrm{v}$ prvi vrsti namiguje na politične interpretacije, vendar načini, ki konflikt prikazujejo, odpirajo tudi druge interpretacije: antropološke, etične, psihološke. Ne da bi zaobšli možnost nadčasovnih pomenov, vzpostavlja predelava Sofoklejeve tragedije, ki jo ponuja Valeria Parella (Antigone, 2012), neposredno soočenje s sedanjostjo. Aktualizacijske komponente ne najdemo zgolj v zamenjavi motiva prepovedanega pokopa z motivom zakonske prepovedi evtanazije, temveč se kaže zlasti v dokaj kritičnem nazoru o sodobni zgodovini. Avtoričina pozornost se ustavi na brezciljnem obdobju in na neaktivnem zavedanju javnosti. Poziv njene Antigone - ki ga oblikuje tudi s pomočjo semiotike pogleda in gibanja - $\mathrm{k}$ iskanju občutka odgovornosti in $\mathrm{k}$ delovanju, k iskanju poti iz obdobja stagnacije, ima jasno izraženo etično ter družbeno politično pomembnost. Dvomljivi pa so zlasti izrazit koncept resnice, ki prežema diskurz, in celo preveč eksplicitni načini, s katerimi se ta poziv uresničuje: junaki težijo $\mathrm{k}$ poenostavitvi, funkcija besed pa je omejena na sredstvo komunikacije, medtem ko bi od literarnega besedila pričakovali tudi kaj drugega, posebno pa to, da bi spodbudil refleksijo o kompleksnosti. 


\section{Patrizia Farinelli}

\section{A Critical View on an Aimless Period: Valerie Parrella's Antigone}

Keywords: Antigone, modern versions, epistemology, semiotics of sight, responsibility

The density of motifs in the myth of Antigone could itself explain the reason for such a large number of interpretations and modernized versions of the myth. In these the conflict between the inner voice of the individual and the law of the state is placed at the very centre, thus primarily indicating a political interpretation; however, the ways in which the conflict are shown also allow for other interpretations: anthropological, ethical, psychological ones. Without circumventing the possibility of timeless meanings, Valeria Parella's re-working of the Sophocles tragedy (Antigone, 2012) represents a direct confrontation with the present. The updated elements are to be found not only in the replacing of a forbidden burial with the motif of illegal euthanasia; rather, they manifest themselves especially in the rather critical view of contemporary history. The author's attention is directed at an aimless period and the public's inactive awareness. Her Antigone - which is formed also by means of the semiotics of sight and movement - is a call to seek out a sense of responsibility and a call to action, to seeking a way out of a period of stagnation, and it possesses a clearlyexpressed ethical and social political importance. Dubious is the very powerfully expressed concept of truth which permeates the discourse, and even the overly explicit means with which this call is realised: the heroes have a tendency to simplification, and the function of words is limited merely to a means of communication, even though one would expect something else from a literary text, that it might prompt special reflection on complexity. 